\title{
The Coulombic Nature of the van der Waals Bond Connecting Conducting Graphene Layers in Graphite*
}

\author{
Raji Heyrovska1,2 \\ ${ }^{1}$ Private Research Scientist, Prague, Czech Republic \\ ${ }^{2}$ Former: Academy of Sciences of the Czech Republic, Prague, Czech Republic \\ Email: rheyrovs@hotmail.com \\ Received 29 January 2016; accepted 23 February 2016; published 26 February 2016 \\ Copyright (C) 2016 by author and Scientific Research Publishing Inc. \\ This work is licensed under the Creative Commons Attribution International License (CC BY). \\ http://creativecommons.org/licenses/by/4.0/ \\ c) (i) Open Access
}

\begin{abstract}
Carbon forms a variety of compounds with single, double, triple and the intermediate resonance bonds with atoms of its own or other kinds. This paper is concerned with graphite, a very useful material, which is a stack of electrically conducting graphene layers held together by weak van der Waals (vdW) bonds. It crystallizes in hexagonal and rhombohedral forms, in which the hexagon inter-planar bond distance is $0.34 \mathrm{~nm}$. Here a new and simple approach accounts for this bond length and shows the coulombic nature of the vdW bond.
\end{abstract}

\section{Keywords}

Carbon, Graphite, Graphene, van der Waals Bond, Bond Length, Golden Ratio Based Ionic Radii

Dedicated to geo-carbon expert, Prof. Gustaf Arrhenius, of Scripps Institution of Oceanography, CA

\section{Introduction}

Carbon [1] is a wonder element of Nature. One of its forms, graphite [1] [2], crystallizes in hexagonal and rhombohedral forms. It is a solid stack of layers of graphene [1] [3] with the inter-planar spacing of $0.34 \mathrm{~nm}$ in both forms [1] [2], held by weak van der Waals (vdW) bonds [4]. This short article provides for the first time a new and simple interpretation of the length and nature of this bond.

\section{Atomic Structure of Graphene}

Graphene is a two-dimensional network of regular hexagons of identical carbon atoms, with equal inter-atomic

*A preliminary report of this work as preprint is at: http://www.vixra.org/abs/1601.0273. 
spacing of $\mathrm{d}(\mathrm{CC})_{\mathrm{res}}=0.142 \mathrm{~nm}$ [1]-[3] [5]-[7]. It is usually represented like a wire mesh in the ball and stick model [3]. Its electrical conductivity as an atomically thin material makes it very useful in many ways, see e.g., [8]. Its structure at the atomic level was worked out by the author [5]-[7], in which all the details can be found. The Figure 4 in [7] shows the alternately charged cations and anions, which are the ionic resonance forms (as for the $\mathrm{H}_{2}$ molecule [2]) of the adjacent $\mathrm{C}$ atoms bound by covalent bonds, responsible for electrical conduction in graphene. This is shown in Figure 1 (a) here. The sum of the radii of the cation $\mathrm{R}(+)$ and anion $\mathrm{R}(-)$ is equal to the covalent bond length, d(CC), [7] [9]. The atomic and ionic radii in Figure 1(a) are related by the Golden ratio, $\varphi=\left(1+5^{1 / 2}\right) / 2=1.618$ as explained in [7] [9] and in the legend for Figure 1. The covalent radius, $R_{\text {Cres }}=\mathrm{d}(\mathrm{CC})_{\mathrm{res}} / 2$ $=(\varphi-1 / 2) \mathrm{a}_{\mathrm{B}}=0.71 \mathrm{~nm}$, where $\mathrm{a}_{\mathrm{B}}$ is the Bohr radius obtained from the first ionization potential [10] [11].

\section{Atomic Structure of Graphite}

In graphite [1] [2], the graphene layers are connected by weak vdW bonds. The vdW bonds exist in a variety of molecules [12] and nano-structures [13]. These bonds are, in general, longer than the covalent bonds. Pauling [2] describes vdW bonds as arising out of the attractive and repulsive forces, and defines the vdW radius as half the corresponding bond length. In the case of carbon, the reported value of vdW radius is around $0.17 \mathrm{~nm}$ [4] [10] [12]. The unit cell of graphite [1] has the lattice constants, $a=0.246 \mathrm{~nm}$ and $\mathrm{c}=0.671 \mathrm{~nm}$. The inter-planar spacing in (both hexagonal and rhombohedral) graphite is $\mathrm{c} / 2=0.336 \mathrm{~nm}$, which is the length of the vdW bond. Half of this is the van der Waals radius, $\mathrm{R}_{\mathrm{vdw}}=0.168 \mathrm{~nm}$.

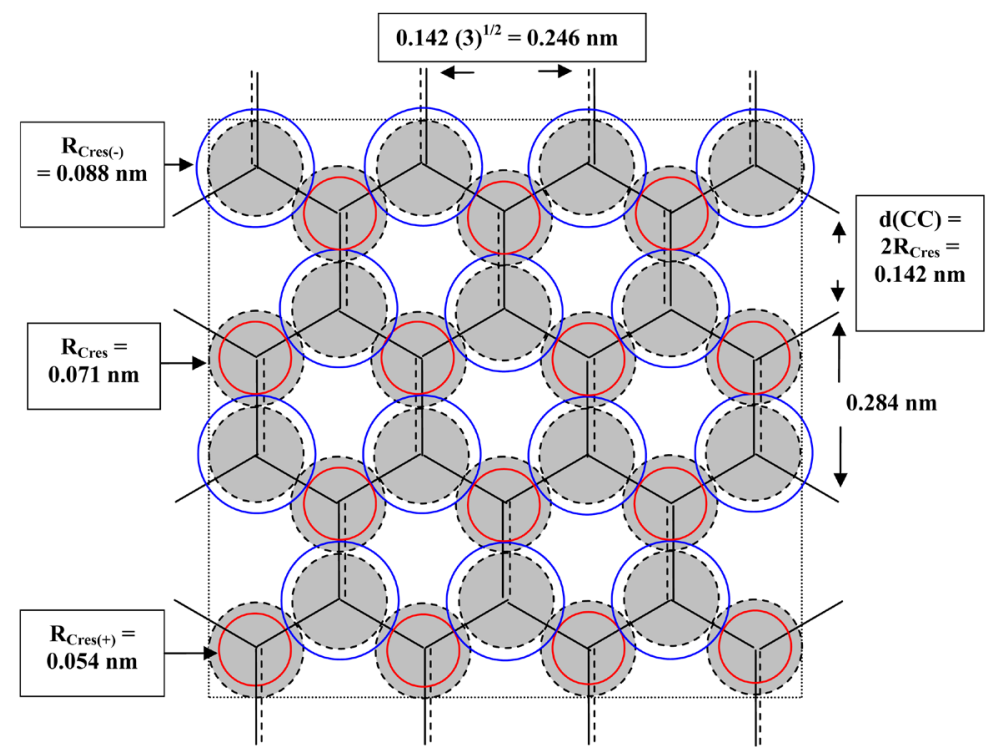

(a)

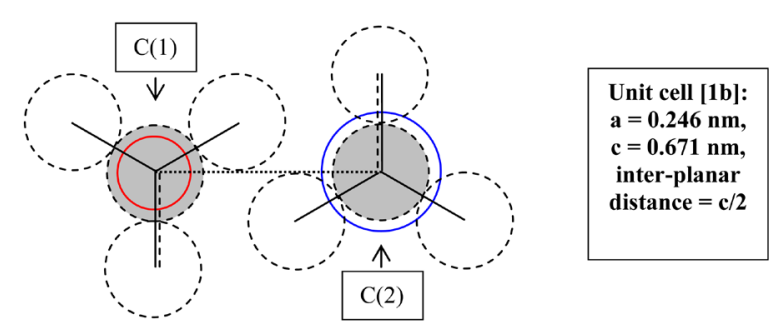

$\mathrm{d}[\mathrm{C}(1) \ldots \mathrm{C}(2)]=\mathrm{a}+\mathrm{R}_{\text {Cres(-) }}=0.246+0.088=0.334 \mathrm{~nm}=$

inter-planar distance $(c / 2)=$ van der Waals bond length

(b)

Figure 1. (a) Graphene [7]. Bond length $d(C C)=2 R_{\text {Cres }}=0.142 \mathrm{~nm}$. The alternate red and blue circles are cations and anions (responsible for conduction) of radii, $\mathrm{R}_{\mathrm{Cres}(+)}=\mathrm{d}(\mathrm{CC}) / \varphi^{2}$ and $\mathrm{R}_{\mathrm{Cres}(-)}=\mathrm{d}(\mathrm{CC}) / \varphi$ and $\mathrm{d}(\mathrm{CC})=$ $\mathrm{R}_{\mathrm{Cres}(+)}+\mathrm{R}_{\mathrm{Cres}(-)}$, where $\varphi$ is the Golden ratio (see [7] for details); (b) Graphite. Atoms C(1) and C(2) are in adjacent graphene layers connected by the van der Waals bond as shown by the dotted line. 


\section{The van der Waals Bond in Graphite}

In Figure 1(b) are shown two carbon atoms, C(1) and C(2) in two adjacent graphene layers (1) and (2) connected by the vdW bond of length $\mathrm{d}(\mathrm{C} . . \mathrm{C})_{\mathrm{vdW}}=2 \mathrm{R}_{\mathrm{vdW}}=\mathrm{c} / 2=0.336 \mathrm{~nm}$. On examining this distance, $\mathrm{c} / 2$, it was astonishing to find that it is actually the sum of the lattice constant, a, which is the width, $3^{1 / 2} \mathrm{~d}(\mathrm{CC})_{\mathrm{res}}$, of the graphene hexagon $(0.246 \mathrm{~nm}$, see Figure $1(\mathrm{a}))$ and the anionic radius, $\mathrm{R}_{\mathrm{C}_{(-)}}=0.088 \mathrm{~nm}$ of carbon,

$$
\mathrm{d}(\mathrm{C} . . \mathrm{C})_{\mathrm{vdW}}=\mathrm{c} / 2=\mathrm{a}+\mathrm{R}_{\mathrm{C}(-)}=\mathrm{d}(\mathrm{CC})_{\text {res }}\left(3^{1 / 2}+1 / \varphi\right)=0.246+0.088=0.334 \mathrm{~nm}
$$

where, $\mathrm{d}(\mathrm{CC})_{\text {res }}=0.142 \mathrm{~nm}, \mathrm{a}=0.142\left(3^{1 / 2}\right)=0.246 \mathrm{~nm}$ and $\mathrm{R}_{\mathrm{C}(-)}=0.142 / \varphi=0.088 \mathrm{~nm}$. It can be seen that all the distances are related to the Golden ratio, $\varphi$ [7] [9] [11].

This shows, for the first time, that the vdW bond in graphite is a long range coulombic bond between the positively charged carbon cation, $\mathrm{C}(1)(+)$ on graphene layer (1) and the negatively charged carbon anion , $\mathrm{C}(2)(-)$ in layer (2), separated by the distance, $\mathrm{a}+\mathrm{R}_{\mathrm{C}(-)}$.

\section{Discussion}

Graphene, a hexagonal network of identical carbon atoms, has become the wonder material of attention for its many useful properties [3] [8] [14]. Its structure at the atomic level was established by the author [5]-[7] based on the discovery [9] that atomic and/or ionic radii are additive in chemical bonds in small as well as large molecules. For a power point review talk, see [15]. The electrical conduction in graphene was attributed [7] to the alternate positively and negatively charged carbon atoms in the ionic resonance forms (as for $\mathrm{H} 2$ molecule [2]). As described in [7] [9] [15], the exact radii $\mathrm{R}(+)$ and $\mathrm{R}(-)$ of these ionic forms could be obtained as the Golden sections of the interatomic distance in graphene, $d(C C)=0.142 \mathrm{~nm}$. Graphite is a stack of graphene layers (crystallizing in hexagonal and rhombohedral forms depending on the conditions [16]) bound by vdW bonds [1] [2] [4]. Although the inter-planar distance, $C(1) . . C(2)=0.336 \mathrm{~nm}$ (see Figure $1(\mathbf{b})$ ) held by the vdW forces is known [4] [12], a simple exact interpretation of this bond in graphite had been lacking. In this paper, it is shown for the first time that the known vdW bond length is the sum of the width of graphene hexagon (=a, the unit cell parameter of graphite) and the anionic radius, $\mathrm{R}(-)$. This means that the vdW bond is of coulombic nature and is the distance between the $\mathrm{C}(1)(+)$ cation and $C(2)(-)$ anion as shown in Figure $1(b)$. This work accounts for the first time why graphite is a conductor and finds its use as an electrode. For the coulomb drag in graphene layers at longer distances of $50 \mathrm{~nm}$ and $100 \mathrm{~nm}$, and for scaling laws for van der Waals interactions in nanostructured materials, see [17] [18] respectively.

\section{Acknowledgements}

The author is grateful to the Editor and reviewers for their useful comments and to SCIRP for partial financial assistance towards APC.

\section{References}

[1] https://en.wikipedia.org/wiki/Carbon, 2016. https://en.wikipedia.org/wiki/Graphite, 2016. https://en.wikipedia.org/wiki/Graphene, 2016.

[2] Pauling, L. (1960) The Nature of the Chemical Bond. Cornell University Press, New York.

[3] The Home of Graphene (2016). http://www.graphene.manchester.ac.uk/

[4] https://en.wikipedia.org/wiki/Van_der_Waals_force, 2016. https://en.wikipedia.org/wiki/Van_der_Waals_radius, 2016.

[5] Heyrovska, R. (2008) Atomic Structures of Graphene, Benzene and Methane with Bond Lengths as Sums of the Single, Double and Resonance Bond Radii of Carbon. http://arxiv.org/ftp/arxiv/papers/0804/0804.4086.pdf

[6] Heyrovska, R. (2008) Various Carbon to Carbon Bond Lengths Inter-Related via the Golden Ratio, and their Linear Dependence on Bond Energies. arXiv:0809.1957

[7] Heyrovska, R. (2010) Bonding Distances as Exact Sums of the Radii of the Constituent Atoms in Nanomaterials-Boron Nitride and Coronene. http://arxiv.org/ftp/arxiv/papers/1004/1004.2667.pdf

Heyrovska, R., Atchison, L. and Narayan, S. (2010) Precsie Atomic Structures of Three Novel Nanomaterials in Nanotechnology, Biomedicine and Cosmology: Graphene, Boron Nitride and Coronene. The Nanomaterials Symposium, 
JHU/APL Kossiakoff Center, MD. http://precedings.nature.com/documents/4357/version/1

[8] Science News (2015) New Design Points a Path to the 'Ultimate’ Battery. ScienceDaily. http://www.sciencedaily.com/releases/2015/10/151029152629.htm

[9] Heyrovska, R. (2005) The Golden Ratio, Ionic and Atomic Radii and Bond Lengths. Molecular Physics, 103, 877-882. http://dx.doi.org/10.1080/00268970412331333591

[10] Heyrovska, R. (2008) Direct Dependence of Covalent, van der Waals and Valence Shell Radii of Atoms on Their Bohr Radii for Elements of Groups 1A - 8A. Philippine Journal of Science, 137, 133-139.

[11] Heyrovska, R. (2013) Atomic, Ionic and Bohr Radii Linked via the Golden Ratio for Elements Including Lanthanides and Actinides. International Journal of Sciences, 2, 63-68. http://www.ijsciences.com/pub/pdf/V2-201304-18.pdf

[12] Alvarez, S. (2013) A Cartography of the van der Waals Territories. Dalton Transactions, 42, 8617-8636. http://dx.doi.org/10.1039/c3dt50599e

[13] Geim, A.K. and Grigorieva, I.V. (2013) Van der Waals Heterostructures. Nature, 499, 419-425. http://dx.doi.org/10.1038/nature12385

[14] http://www.graphenea.com/pages/graphene-properties\#.VqtNEY-cEb4.

[15] Heyrovska, R. (2015) The Golden Ratio, a Key Geometrical Constant in Atomic Architecture. Proceedings of the 113th Statistical Mechanics Conference, Piscataway, 10-12 May 2015, Program of Conference, Abstract B2, 22. https://www.researchgate.net/publication/281270357

[16] Arrhenius, G. (2016) Personal Communication.

[17] D’Amico, I. and Sharapov, S.G. (2013) Universal Scaling of Coulomb Drag in Graphene Layers. http://arxiv.org/pdf/cond-mat/0410769v1.pdf

[18] Gobre, V.V. and Tkatchenko, A. (2013) Scaling Laws for van der Waals Interactions in Nanostructured Materials. Nature Communications, 4, 2341. http://www.nature.com/ncomms/2013/130819/ncomms3341/full/ncomms3341.html\#f2 